\title{
Liquid-crystalline blue phase III and structures of broken icosahedral symmetry
}

\author{
Lech Longa, ${ }^{*}$ Werner Fink, and Hans-Rainer Trebin \\ Institut für Theoretische und Angewandte Physik, Universität Stuttgart, D-70550 Stuttgart, Pfaffenwaldring 57, Germany
}

(Received 25 November 1992)

\begin{abstract}
The structure of the liquid-crystalline blue phase III (BPIII) is still unknown and remains one of the mysteries of liquid-crystal physics. We take all icosahedral space-group symmetries of the reciprocal space for BPIII and study their thermodynamic stability within the frame of an extended de Gennes-Ginzburg-Landau free-energy expansion. The stability of the icosahedral structures is compared with that of the cholesteric phase and of the cubic blue phases. Strikingly, even though the extended model contains three extra parameters, we could not detect a region of parameter space where icosahedral structures are absolutely stable just below the isotropic phase.
\end{abstract}

PACS number(s): 64.70.Md, 05.70.Ce, 61.30.-v, 61.50.Em

Chiral nematic liquid crystals show strange structures in a narrow temperature range $(\sim 0.1-2.5 \mathrm{~K})$ between the standard isotropic liquid phase (ISO) and the cholesteric phase (C), known as blue phases $[1,2]$. Some compounds exhibit three different blue phases, labeled BPI, BPII, and BPIII with ascending temperature.

Experiments conducted on a large variety of materials prove that BPI and BPII have body-centered-cubic structure with a space-group symmetry of $O^{8}\left(I 4_{1} 32\right)$ and simple cubic structure with space-group symmetry $\mathrm{O}^{2}\left(\mathrm{P}_{2} 32\right)$, respectively. But unlike ordinary crystals, where three-dimensional periodicity is formed by a long-range positional order of the centers of mass of the molecules, in blue phases the constituent molecules are positionally disordered. The three-dimensional cubic order is established here by the mean molecular orientations yielding sharply peaked circularly polarized Bragg reflections of visible and UV light. The structure of the third blue phase, BPIII, which is formed directly on cooling the isotropic liquid, is not known at present. Contrary to BPI and BPII, BPIII does not exhibit Bragg scattering of light, but instead a broad selective reflection band typical of an amorphous system $[1,3,4]$. BPIII transforms into an ordinary isotropic liquid phase by a first-order transition with a transition enthalpy $\Delta H \approx 170 \mathrm{~J} / \mathrm{mol}$ for cholesteryl nonanoate [5]. The transformation into the lower temperature BPI (or BPII) is also first order but about two orders of magnitude weaker (i.e., $\Delta H \approx 1.9 \mathrm{~J} / \mathrm{mol}[6]$ ). These data indicate that the order in BPIII must be close to that in BPI and BPII [7].

Currently there are three different models for the structure of BPIII: (a) Amorphous model [1]. Here BPIII is viewed as a collection of local objects (double twist cylinders, domains of BPI or BPII, domains of body-centeredcubic $O^{5}$ structure, etc.), which are randomly floating in a sea of an isotropic phase. An important element of this model is that the difference between the isotropic liquid and BPIII lies basically in the local structure. (b) Model of bond-orientational order [8]. Melting of BPI and BPII lattices towards an ordinary isotropic liquid could proceed via an intermediate isotropic liquid with a residual long-range orientational order, characterized by nonzero bond order parameters $\left\langle Y_{m}^{4}\right\rangle,\left\langle Y_{m}^{6}\right\rangle$, etc. Such a mechanism was long ago envisaged by Nelson and Toner [9] and is known as defect mediated melting. For BPIII it would be a "defect mediated melting of a periodic lattice of disclinations." Unfortunately until now no experiments have been carried out to test the existence of residual order.

For all their attractiveness, it is as yet not clear how to incorporate the ideas expressed by the above two models into a thermodynamically consistent theoretical scheme. The only currently existing model of BPIII that allows for systematic studies is the so-called (c) Icosahedral model [10]. It assumes a very intriguing possibility for BPIII, namely, that it possesses a quasiperiodic symmetry of an icosahedral "liquid" quasicrystal [11].

Generally, many experimental data seem consistent with all three models, although there are two "problems" with the icosahedral one. First, the existence of icosahedral symmetry implies that Bragg scattering of light should show a pattern similar to x-ray scattering of quasicrystalline metallic alloys, such as Al-Mn [12]. A quasicrystalline pattern has not been found in BPIII [13]. According to Hornreich [10] this might be due to a destructive character of phasons, which may prevent the growing of single quasicrystallites with a size of several microns, necessary to resolve closely positioned icosahedral peaks.

The second problem is connected with structural stability calculations based upon the standard de GennesGinzburg-Landau (deGL) theory of liquid crystals [14]. The deGL theory has proved extremely successful in describing BPI and BPII phases, in reproducing gross features of the corresponding phase diagrams $[1,14]$, and in studying the effect of external electric or magnetic fields on blue phases [12]. When applied to quasicrystalline models, however, it shows that in physically relevant chirality ranges the quasicrystalline order is always less stable than the cholesteric and the cubic structures involved.

Is then BPIII an icosahedral, bond oriented or orientationally amorphous structure? At present it is difficult to answer this question unambiguously, and it seems that 
any calculations in favor of (or against) one of the three models are crucial. In that respect the icosahedral model, which could be examined systematically, is the most interesting one. There are also several other reasons that make the icosahedral model worth further studies: (1) "Intrinsic inaccuracy" of the deGL model. The deGL theory certainly cannot be fully correct, for it predicts that in the physically relevant range of chiralities, BPIII is characterized by a body-centered-cubic space group $O^{5}$. The latter, however, has never been detected experimentally in chiral systems. The deGL model also predicts no phase transition between ISO and BPI phases, which remains in contrast to experiment. These results indicate the necessity of generalizing the deGL model. (2) Icosahedral BPIII and the role of bulk terms. Within the scheme proposed by Hornreich (see, e.g., [14]) there are two possible directions in which the deGL model could be generalized. One way is to include higher-order bulk terms in the original expansion. Since the transitions involving blue phases are first order, these higher-order terms in the dielectric tensor order parameter $\mathbf{Q}$ may play an important role. Actually this has already been demonstrated for uniaxial nematics in the vicinity of the nematic-isotropic phase transition [15]. As shown in [15] the experimental data for nematic liquid crystals fit very well to the model, with a sixth-order term being dominant. If the presence of chirality does not change the phenomenological parameters significantly, then one may expect for chiral systems the existence of a new physically relevant sector in the extended parameter space, which is not accessible within the standard model [14]. We shall follow this idea in the present Brief Report. (3) Exceptional role of phasons in the icosahedral BPIII. While in ordinary crystals Goldstone excitations are associated with three acoustic phonons, in quasicrystalline matter there exist three additional Goldstone modes, called phasons. If the quasiperiodic concept is relevant to understanding BPIII, then the role played by phasons must be quite exceptional. Unlike with metallic quasicrystals, where phasons are pinned to form a quenched positional disorder, in liquid BPIII they could be mobile, yielding an annealed orientational (background) disorder. This might provide an additional channel for relaxation processes [16]. It could also suppress growth of large-dimension single quasicrystallites. Furthermore, the growth of quasicrystalline domains of any size is no longer controlled by the boundary surface. (4) Shortrange icosahedral order. If the icosahedral representation of BPIII structure is not a precise one, it could serve as a starting point to construct more realistic models. As discussed by Alexander and McTague [17], even in amorphous matter, the local icosahedral symmetries are strongly favored.

From the analysis as given it follows that the possibility of characterizing BPIII by an icosahedral structure is indeed extremely intriguing and certainly deserves further considerations. This Brief Report communicates results of a systematic stability analysis for a large class of quasiperiodic structures of BPIII within the framework of an extended de Gennes-Ginzburg-Landau theory (EdeGL), where higher-order bulk terms are added.
Free energies of icosahedral structures are compared with those of C, BPI, BPII, and $O^{5}$ structures.

To outline the basic ideas let us recall that the deGL theory is based upon the free-energy expansion in terms of the traceless part $\mathbf{Q}(\mathbf{r})$ of the dielectric tensor field. By definition, $\mathbf{Q}(\mathbf{r})$ vanishes for locally disordered, $\mathrm{SO}(3)$ symmetric configurations and becomes nonzero if the local orientational order is characterized by $D_{\infty}$ or $D_{2}$ symmetries. The expansion is usually taken to the lowest order in $\partial_{\mu} \mathbf{Q}$ and to fourth order in $\mathbf{Q}$, which ensures thermodynamic stability for all temperatures and chiralities. Here we consider a more general expansion of the free energy of chiral liquid crystals that is complete up to sixth order in $Q_{\alpha \beta}$ [18]. In the units of Grebel, Hornreich, and Shtrikman [14] it reads

$$
\begin{aligned}
F=\frac{1}{v} \int \mathrm{d}^{3} \mathbf{x}\{ & \frac{1}{4}\left[t \operatorname{tr} \mathbf{Q}^{2}-2 \kappa \epsilon_{i j k} Q_{i n} Q_{j n, k}+\left(Q_{i j, l}\right)^{2}\right] \\
& -\sqrt{6} \beta \operatorname{tr} \mathbf{Q}^{3}+\gamma\left(\operatorname{tr} \mathbf{Q}^{2}\right)^{2} \\
& -\frac{1}{\sqrt{6}} \phi\left(\operatorname{tr} \mathbf{Q}^{2}\right)\left(\operatorname{tr} \mathbf{Q}^{3}\right) \\
& \left.+\frac{1}{6} \epsilon\left(\operatorname{tr} \mathbf{Q}^{2}\right)^{3}+\frac{1}{6} \epsilon^{\prime}\left[\left(\operatorname{tr} \mathbf{Q}^{2}\right)^{3}-6\left(\operatorname{tr} \mathbf{Q}^{3}\right)^{2}\right]\right\},
\end{aligned}
$$

where $t$ is the reduced temperature, $\kappa$ is the chirality parameter, and where $\epsilon^{\prime}>-\epsilon$ is required for stability. [An additional term, proportional to $\left(Q_{i j, i}\right)^{2}$, vanishes for the C, cubic BP, and for the BPIII structures being presented here.] Two out of five material parameters $\left(\beta, \gamma, \phi, \epsilon, \epsilon^{\prime}\right)$ are redundant [14] and can be set 0 or \pm 1 . The $(\kappa, t)$ phase diagrams are thus parametrized by an extra three parameters. The case studied by Grebel, Hornreich, and Shtrikman [14], corresponds to the assignment $\beta=1, \gamma=1 ; \phi=\epsilon=\epsilon^{\prime}=0$.

Note that for locally uniaxial structures the sixthorder term, weighted by $\epsilon^{\prime}$, vanishes. It becomes greater than zero in the presence of locally biaxial configurations. Thus for $\epsilon^{\prime}>0$ the model represented by Eq. (1) characterizes locally uniaxial liquid-crystalline materials, while for $-\epsilon<\epsilon^{\prime}<0$ it describes liquid crystals of biaxial molecules. The ratio $w$ of the sixth order terms: $w=\int d^{3} \mathbf{x}\left[\left(\operatorname{tr} \mathbf{Q}^{2}\right)^{3}-6\left(\operatorname{tr} \mathbf{Q}^{3}\right)^{2}\right] / \int \mathrm{d}^{3} \mathbf{x}\left(\operatorname{tr} \mathbf{Q}^{2}\right)^{3}$ could be regarded as a measure of phase biaxiality $(0 \leq w \leq 1)$. For purely uniaxial phases $w$ equals 0 , while for phases of maximal biaxiality $w$ equals 1 .

Our objective now is to minimize $F[\mathbf{Q}(\mathbf{r})]$ for arbitrary $(\kappa, t)$ and for fixed values of the material parameters. As the global minimization in the presence of the chiral term $(\kappa \neq 0)$ is not possible, the approach taken is to consider individually all relevant periodic and quasiperiodic structures. For that it is convenient to introduce a parametrization of the field $\mathbf{Q}$ that agrees with the space-group symmetries of the structures considered by expanding $\mathbf{Q}$ into plane waves of helicity,

$\mathbf{Q}(\mathbf{r})=\sum_{* \mathbf{k}} \frac{1}{\sqrt{N_{*} \mathbf{k}}}\left\{\sum_{\mathbf{k} \in *_{\mathbf{k}}}\left[\sum_{m=-2}^{2} Q_{m}(\mathbf{k}, \mathbf{r})\right] \mathbf{e}_{m, \hat{\mathbf{k}}}^{[\mathbf{2}]}\right\}$, 
where

$Q_{m}(\mathbf{k}, \mathbf{r})=Q_{m}(|\mathbf{k}|) \exp \left(\mathrm{i} \mathbf{k} \cdot \mathbf{r}-\mathrm{i} \psi_{m, \hat{\mathbf{k}}}\right)$.

Here $\mathbf{k}$ is taken out of a reciprocal lattice of a space group $G$, where ${ }^{*} \mathbf{k}=\left\{\mathbf{k}^{\prime}: \mathbf{k}^{\prime}=S \mathbf{k},\{S \mid \mathbf{t}\} \in G\right\}$ is the star of $\mathbf{k} ; N_{* \mathbf{k}}$ is the number of prongs of the $\operatorname{star}^{*} \mathbf{k}$, $Q_{m}(|\mathbf{k}|)$ are the variational parameters in the expansion, and finally $\mathbf{e}_{m, \hat{\mathbf{k}}}^{[\mathbf{2}]}$ are the spin $L=2$ tensors represented in an orthogonal, right-handed local coordinate system with $\hat{\mathbf{k}}$ as quantization axis (for a precise definition see $[14,18])$. The selection of the wave vectors $\mathbf{k}$ and the relative phases $\psi_{m, \hat{\mathbf{k}}}$ is fixed by the space-group symmetry $G$ of the structure. The minimization over fields $\mathbf{Q}(\mathbf{r})$ is thus reduced to a minimization over the real amplitudes $Q_{m}(|\mathbf{k}|)$. In practical calculations we restricted the expansion (2) to two leading stars $\left({ }^{*} \mathbf{k}_{1},{ }^{*} \mathbf{k}_{2}\right)$ of the $\mathbf{k}$ vectors. For each ${ }^{*} \mathbf{k}$ we selected $m=2$ modes, which correspond to the low-lying branch of the excitation spectrum of the quadratic part of the free energy [14]. Note that the fifth-order term in the expansion (1b), which counts the closed five-polygons of $\mathbf{k}$ vectors may become quite large for icosahedral symmetries (see Table I).

We start the analysis by discussing the simpler case of the high chirality limit $(\kappa \gg 1)$. In this limit any structure that minimizes the free energy (1) is a linear combination of $m=2$ modes, associated with $\mathbf{k}$ vectors of fixed moduli. For periodic and quasiperiodic structures this means that the free energy is reduced to a polynomial of a single variable $Q_{2}\left(\left|\mathbf{k}_{1}\right|=\right.$ const) (single ${ }^{*} \mathbf{k}_{1}$ approximation). In Table I we list the coefficients of such polynomials for C, BPI, BPII, and for all icosahedral space groups with ${ }^{*} \mathbf{k}_{1}$ forming 30 edges of a regular icosahedron. We also list the biaxality parameter $w$, which in this case is a fixed number, independent of $Q_{2}(|\mathbf{k}|)$. Interestingly, apart from the $5_{3} 32$ structure all other icosahedral structures are strongly biaxial. The least biaxial is the $O^{5}$ $\left(\equiv O^{2}\right.$ ) structure. The strong biaxiality of the icosahedral structures can reduce their free energy appreciably in the biaxial parameter regime $\epsilon^{\prime}<0$. Indeed, the detailed minimization of the free energy for $\epsilon^{\prime}<0$ shows that the icosahedral phases may become more stable than the cubic ones, but they are always less stable than the cholesteric phase, which in this limit is maximally biaxial. In the uniaxial regime $\left(\epsilon^{\prime} \geq 0\right)$ either $O^{5}$ or $O^{8}$ are the most stable structures. Between one of these cubic structures and the low-temperature cholesteric phase the icosahedral phases $F(\equiv B) 5_{n} 32(n=1,3,4)$ can be sta- bilized. Unexpectedly, we found that in the high chirality limit there exists no parameter window in which an icosahedral structure could be absolutely stable just below the isotropic phase.

Given this result one may immediately ask whether an icosahedral structure could be stabilized at physically relevant chiralities. To answer this question we have performed stability calculations for all the structures discussed so far using two stars of wave vectors in the expansion, Eq. (2). A key criterion in selecting a supplementary star ${ }^{*} \mathbf{k}_{\mathbf{2}}$ for icosahedral structures was to require a maximal number of triangles with the original star ${ }^{*} \mathbf{k}_{1}$. An additional requirement was to keep the ratio $\left|\mathbf{k}_{2}\right| /\left|\mathbf{k}_{1}\right|$ close to 1 . Observing correctly the selection rules we arrived at six different icosahedral structures. Five of these structures are associated with the face-centered space-group symmetries $F(\equiv B) 5_{n} 32(n=0, \ldots, 4)$ in reciprocal space [19] with the ratio $\left|\mathbf{k}_{2}\right| /\left|\mathbf{k}_{1}\right| \approx 1.17$. The last one corresponds to a primitive $P_{2} 32$ symmetry with $\left|\mathbf{k}_{2}\right| /\left|\mathbf{k}_{1}\right| \approx 0.95$. For all space groups involved, the polynomial form of the free energy in terms of $Q_{2}\left(\left|\mathbf{k}_{1}\right|\right)$ and $Q_{2}\left(\left|\mathbf{k}_{2}\right|\right)$ was found by combining group-theoretical methods, computer algebra, and numerical techniques. The resulting long formulas together with a more detailed exposition will be published elsewhere. The main results can be summarized as follows: In the regime of the parameters close to that studied earlier by Hornreich and others $[10]\left[\phi, \epsilon, \epsilon^{\prime}<1, \operatorname{sgn}(\gamma)>0\right.$ in Eq. (1)], we recovered the known results with the icosahedral structures being absolutely unstable in the physically relevant chirality interval. For the case when $\epsilon$ becomes dominant and $\gamma$ negative the stability of the $F 5_{n} 32(n=1,3,4)$ is enhanced; this is due to the fourth- and the fifth-order terms in the free-energy expansion. Generally the $F 5_{n} 32$ $(n=1,3,4)$ could be made stable over $O^{8}, O^{2}$ and the cholesteric phase. However, they become unstable when the $O^{5}$ cubic structure is allowed. With the $O^{5}$ structure suppressed an icosahedral phase may appear in exactly this phase diagram regime where normally BPIII is stabilized (see [2]).

In the biaxial regime we recovered the results similar to those of the high chirality limit. Namely, we could stabilize the icosahedral structure with respect to the cubic structures (including $O^{5}$ ), but not with respect to the cholesteric one.

In conclusion, we did not find a direct phase transition between the isotropic liquid and an icosahedral structure within the EdeGL theory and for moduli of the param-

TABLE I. High-chirality-limit bulk contributions to the free energy for various symmetry-allowed structures. Contribution proportional to $\epsilon$ equals $[\epsilon]$.

\begin{tabular}{cccccc}
\hline \hline Structure & {$[\beta]$} & {$[\gamma]$} & {$[\phi]$} & {$[\epsilon]$} & $w$ \\
\hline Cholesteric & 0.000 & 1.000 & 0.000 & 0.167 & 1.000 \\
$O^{5}$ & -1.017 & 1.300 & -0.245 & 0.315 & 0.068 \\
$O^{8}$ & -0.625 & 1.169 & -0.154 & 0.255 & 0.363 \\
$F 532$ & -0.438 & 1.200 & -0.115 & 0.271 & 0.481 \\
$F 5_{1} 32$ & -0.008 & 1.150 & 0.010 & 0.243 & 0.514 \\
$F 5_{2} 32$ & 0.450 & 1.354 & 0.112 & 0.356 & 0.556 \\
$F 5$ & -0.720 & 1.529 & -0.274 & 0.531 & 0.222 \\
$F 5_{4} 32$ & 0.715 & 1.434 & 0.214 & 0.423 & 0.394 \\
\hline \hline
\end{tabular}


eters less than 10. Taking into account the number of extra parameters involved in the expansion (1) the lack of a direct transition between the isotropic phase and an icosahedral structure is quite striking. However, a conclusion that these calculations rule out the icosahedral model would be premature. First of all, the effect of higher-order spatial derivatives of $\mathbf{Q}$ and of fluctuations may be important. Finally, the higher harmonics of the basic wave vectors may also enhance the stability of the $F 5_{n} 32$ and $P 5_{2} 32$ structures, although the corresponding numerical analysis is a formidable task. The $O^{5}$ phase turns out to be extremely stable in large regions of parameter space due to its extremely small biaxiality parameter $w$. The phase has never been seen in experiment, however, and the decisive question addressed to theory is, what mechanism enlarges the free energy of this special space-group symmetry compared to the other structures?

\section{ACKNOWLEDGMENTS}

This research was supported by the Heraeus Foundation and in part by the Polish Project No. 202549101.
* Permanent address: Department of Statistical Physics, Jagellonian University, Reymonta 4, Kraków, Poland.

[1] For recent reviews, see D. C. Wright and N. D. Mermin, Rev. Mod. Phys. 61, 385 (1989); P. P. Crooker, Liq. Cryst. 5, 751 (1989); R. M. Hornreich and S. Shtrikman, Mol. Cryst. Liq. Cryst. 165, 183 (1988); V. A. Belyakov and V. E. Dmitrienko, Usp. Fiz. Nauk 146, 369 (1985) [Sov. Phys. Usp. 28, 535 (1985)].

[2] D. K. Yang and P. P. Crooker, Phys. Rev. A 35, 4419 (1987).

[3] H. Stegemeyer and K. Bergmann, in Liquid Crystals of One-and Two-Dimensional Order, edited by W. Helfrich and G. Heppke (Springer, Berlin, 1980), p. 161.

[4] M. Marcus, J. Phys. (Paris) 42, 61 (1981).

[5] J. Thoen, Phys. Rev. A 37, 1754 (1988).

[6] It is not known at present as to whether such a small value of the specific heat also persists for the BPIII BPI phase transition and how this value evolves with increasing chirality.

[7] For comparison the transition enthalpies at BPI-C and BPII-BPI transitions are 18 and $5.8 \mathrm{~J} / \mathrm{mol}$, respectively.

[8] P. H. Keyes, Phys. Rev. Lett. 65, 436 (1990); R. L. Rakes, Jr. and P. H. Keyes, Mol. Cryst. Liq. Cryst. 198, 79 (1991).

[9] D. R. Nelson and J. Toner, Phys. Rev. B 24, 363 (1981).

[10] R. M. Hornreich and S. Shtrikman, Phys. Rev. Lett. 56, 1723 (1986); D. S. Rokshar and J. P. Sethna, ibid. 56, 1728 (1986); R. M. Hornreich, in Aperiodicity and Order, Volume 3: Extended Icosahedral Structures, edited by M. V. Jarić and D. Gratias (Academic, New York, 1989), p. 189; V. M. Filev, JETP Lett. 43, 677 (1986) [Pis'ma Zh. Exp. Teor. Fiz. 43, 523 (1986)]; See also H. Kleinert and K. Maki, Fortschr. Phys. 29, 219 (1981).

[11] D. Shechtman, I. Blech, D. Gratias, and J. W. Cahn, Phys. Rev. Lett. 53, 1951 (1984).

[12] R. M. Hornreich, M. Kugler, and S. Shtrikman, Phys. Rev. Lett. 54, 2099, (1985).

[13] D. K. Yang, P. P. Crooker, Liq. Cryst. 7, 411 (1990); H.-S. Kitzerow, P. P. Crooker, S. L. Kowk, and G. Heppke, J. Phys. (Paris) 51, 1303 (1990); G. Heppke, H.-S. Kitzerow, D. Lötsch, and Ch. Papenfuß, Liq. Cryst. 8, 407 (1990); H.-S. Kitzerow, P. P. Crooker, S. L. Kowk, J. Xu, and G. Heppke, Phys. Rev. A 42, 3442 (1990).

[14] (a) H. Grebel, R. M. Hornreich, and S. Shtrikman, Phys. Rev. A 28, 1114 (1983); (b) H. Grebel, R. M. Hornreich, and S. Shtrikman, ibid. 30, 3264 (1984).

[15] See, e.g., F. Gramsbergen, L. Longa, and W. H. de Jeu, Phys. Rep. 135, 195 (1986), and references therein.

[16] It is possible that the relaxation process in BPIII, observed by Yang and Crooker, could be explained in this way $[13]$.

[17] S. Alexander and J. McTague, Phys. Rev. Lett. 41, 702 (1978).

[18] For a general discussion of higher-order expansions in terms of $Q_{\alpha \beta}(\mathbf{r})$, see, e.g., L. Longa and H.-R. Trebin, Phys. Rev. A 39, 2160 (1989); 42, 3453 (1990).

[19] Our icosahedral space group notation is adopted after D. S. Rokhsar, D. C. Wright, and N. D. Mermin, Phys. Rev. B 37, 8145 (1988). 